\title{
Role of Sirtuin 1 in the pathogenesis of ocular disease (Review)
}

\author{
MENGWEN ZHOU ${ }^{1}$, JING LUO $^{1}$ and HUIMING ZHANG ${ }^{2}$ \\ ${ }^{1}$ Department of Ophthalmology, Hunan Clinical Research Center of Ophthalmic Disease, The Second Xiangya Hospital, \\ Central South University; ${ }^{2}$ Department of Dermatology, Hunan Key Laboratory of Medical Epigenomics, \\ The Second Xiangya Hospital, Central South University, Changsha, Hunan 410011, P.R. China
}

Received November 14,2017; Accepted March 29, 2018

DOI: $10.3892 / \mathrm{ijmm} .2018 .3623$

\begin{abstract}
Sirtuin (SIRT)1, a member of the SIRT family, is a highly conserved $\mathrm{NAD}^{+}$-dependent histone deacetylase, which has a regulatory role in numerous physiological and pathological processes by removing acetyl groups from various proteins. SIRT1 controls the activity of numerous transcription factors and cofactors, which impacts the downstream gene expression, and eventually alleviates oxidative stress and associated damage. Numerous studies have revealed that dysfunction of SIRT1 is linked with ocular diseases, including cataract, age-associated macular degeneration, diabetic retinopathy and glaucoma, while ectopic upregulation of SIRT1 protects against various ocular diseases. In the present review, the significant role of SIRT1 and the potential therapeutic value of modulating SIRT1 expression in ocular development and eye diseases is summarized.
\end{abstract}

\section{Contents}

1. Introduction

2. Developmental roles and distributions of SIRT1 in the eye

3. SIRT1 and eye diseases

4. Perspective

\section{Introduction}

Epigenetic alterations have critical roles in various physiological and pathological processes (1). Histone deacetylases (HDACs) are among the most important epigenetic regulators, which deacetylate lysine residues on specific histone and non-histone proteins (2). According to the homologies of the respective

Correspondence to: Dr Huiming Zhang, Department of Dermatology, Hunan Key Laboratory of Medical Epigenomics, The Second Xiangya Hospital, Central South University, 139 Middle Renmin Road, Changsha, Hunan 410011, P.R. China

E-mail: huimingzhang@csu.edu.cn

Key words: sirtuin 1, eye disease, deacetylation, resveratrol, degeneration, angiogenesis yeast orthologues, human HDACs have been divided into four classes $(3,4)$. The human sirtuin (SIRT) family, belonging to class-III HDACs, is a class of $\mathrm{NAD}^{+}$-dependent deacetylases and contains seven members (SIRT1-7) (5). Among them, SIRT1 has been most widely studied. The human SIRT1 protein contains 747 amino acids and is composed of three major regions (Fig. 1) (6,7). The level and activity of SIRT1 is regulated at the transcriptional and post-transcriptional level (8), with post-translational modifications including phosphorylation (9), sumoylation (10), methylation (11), S-nitrosylation (12) and carboxylation (13). Besides, the activity of SIRT1 distinctly depends on the NAD ${ }^{+} \mathrm{NADH}$ ratio and may be affected by nucleocytoplasmic shuttling (14).

The SIRT family has been identified to be highly evolutionarily conserved in various organisms from bacteria to humans (15). SIRT1, maintaining the silenced chromatin state and genomic stability (16), has been associated with various physiological and pathological processes and conditions, including DNA repair, metabolic regulation, aging, oxidative stress, angiogenesis, inflammation, neurodegenerative diseases and cardiovascular dysfunction. Previous reviews have indicated the major roles of SIRT1 in retinal and ocular aging (17-19). Increasing evidence has demonstrated that SIRT1 is also involved in other eye diseases, which are not limited to aging. The present review focuses on the current understanding and the potential therapeutic value of SIRT1 in ocular disorders.

\section{Developmental roles and distribution of SIRT1 in the eye}

It has been reported that mice carrying two null alleles of SIRT1 (also known as SIR2a in lower organisms) are small, and most of them die shortly after birth. Outbred SIRT1-null animals usually survived until adulthood, but were sterile (20). In SIRT1-deficient mice, eyelids remained closed accompanied by abnormalities of the cornea, lens and retina $(20,21)$, and eyes were smaller, with the optic fissure being abnormally closed (22). Furthermore, there were significantly thinner retinal cell layers and disordered inner and outer nuclear layers. In addition, it was difficult to identify the inner and outer segments of photoreceptor cells. These eye defects occurred in early embryos, which implied that SIRT1 regulated ocular morphogenesis and retinal development (22). In addition, SIRT1 was reduced in the retina of mice with knockout of E2fs, which are essential positive cell cycle regulators, resulting in 
hyperacetylation of p53, a pro-apoptotic factor downstream of SIRT1, and increased retinal progenitor cell apoptosis (23). Treatment of pregnant mice with resveratrol, an activator of SIRT1, significantly blocked the apoptosis at P0 (23). Thus, the pro-survival role of E2f/SIRT1/p53 in retinal development has been established.

SIRT1 was detected in the cornea, lens, ciliary body, retinal pigment epithelium (RPE) and neuroretina in mice and humans, and in human normal conjunctival epithelium (24-26). Jaliffa et al (24) firstly reported that SIRT1 was predominantly localized in the nuclei of ocular cell, including corneal epithelial cells, ciliary process cells and ciliary epithelial cells, the epithelial and fiber cells of the lens, RPE cells and melanocytes, and exclusively in the nuclei of cells of the outer nuclear layer (ONL), inner nuclear layer (INL) and ganglion cell layer (GCL) but never in the cytoplasm. They also detected SIRT1 in the cytoplasm of corneal epithelial cells and choroidal vessel endothelial cells (24). Another study reported that SIRT1 was mainly expressed in the cytoplasm in the GCL, inner plexiform layer (IPL), outer plexiform layer (OPL), and inner segments of photoreceptors (26). In addition, SIRT1 was identified to be exclusively expressed in the cytoplasm of mouse retinal progenitor cells (RPCs), while it was present in the nuclei and cytoplasm in human RPCs (26). These apparently different SIRT1 distributions in different cell types of different species suggest that SIRT1 expression may be variable during different periods of retinal development and cell differentiation.

\section{SIRT1 and eye diseases}

SIRT1 and corneal diseases. Under normal conditions, the corneal epithelium is important for the maintenance of the physiological corneal function, rendering the cornea highly resistant to microbial invasion. A high-glucose (HG) environment led to the downregulation of SIRT1 and upregulation of acetylated p53 (Ac-p53) and insulin-like growth factor binding protein-3 (IGFPB3) in primary human corneal epithelial cells as well as corneas from insulin (Ins) $2^{\text {Akita/+ }}$ mice (27). Overexpression of SIRT1 in corneal epithelial cells and Ins2 $2^{\text {Akita/+ }}$ mice significantly led to a downregulation of Ac-p53 and IGFPB3, and an upregulation of the levels of phosphorylated (p)-AKT and IGF-1 receptor precursor. In addition, SIRT1 overexpression in corneal epithelium promoted the wound healing process under HG conditions, which may involve reinforcement of the IGFBP3/IGF-1/AKT pathway with the decrease of Ac-p53 (27). With the progression of diabetic dry eye (DE) in a mouse model, SIRT1 expression in the cornea rose in the first stage and then decreased. Furthermore, the expression of forkhead box $\mathrm{O} 3$ (FOXO3), which ameliorated the response to oxidative stress as a substrate of SIRT1, and the antioxidant enzyme Mn-superoxide dismutase (MnSOD) protein had a similar tendency with SIRT1, which suggests a role of SIRT1 in the resistance to oxidative stress (28). In summary, SIRT1 activation may be an effective approach for treating diabetic keratopathy.

Recent studies have demonstrated that microRNAs (miRNAs) may regulate corneal development and diseases. miRNA-204 directly downregulated SIRT1 in the cornea, and overexpression of miRNA-204 in human corneal epithelial cells inhibited cell cycle progression, cell proliferation and cell migration during the healing of wounded corneal epithelium in mice $(29,30)$. Furthermore, miR-204-5p antagomir promoted the wound healing process via SIRT1 regulation in type 1 diabetic Ins $2^{\text {Akita/+ }}$ mice (30). Wang et al (31) reported that miRNA-182 was the downstream miRNA target of SIRT1 under HG conditions, and protected against peripheral damage of trigeminal ganglions and keratopathy in diabetic $\mathrm{db} / \mathrm{db}$ mice by decreasing the expression of one of its target genes, NADPH oxidase 4 . Therefore, SIRT1 may protect the cornea through the miRNA-mRNA regulatory network.

SIRT1 and cataract. Age-associated cataract (ARC) is a condition characterized by multiple mechanisms and has various risk factors, including genetic, metabolic, nutritional and environmental factors, as well as other ocular diseases (32). Previous studies have indicated that resveratrol is able to protect human lens epithelial cells from oxidative damage induced by $\mathrm{H}_{2} \mathrm{O}_{2}(33,34)$ and suppress experimental cataract formation in rats (35). The SIRT1 levels in the lens were identified to be significantly decreased in individuals aged $\geq 51$ years, and to be negatively correlated with ARC in humans (36). Of note, SIRT1 was significantly increased in patients aged $>50$ years with ARC compared with that in age-matched subjects without ARC (37), and SIRT1 levels in the aqueous humor of ARC patients were positively correlated with the severity of nuclear cataract (38). Furthermore, while the expression of the downstream components of SIRT1, FOXO3a and FOXO4, was downregulated with age, it exhibited relative increases in ARC patients (37). By contrast, the expression of p53 increased with age, but active Ac-p53 was decreased in older patients with cataract compared with that in old individuals without cataract (37). These studies indicate that the increased SIRT1 may function as a compensation to alleviate ARC formation through inhibiting its downstream p53 acetylation and activating the FOXO pathway (37). Another study indicated that the enhanced interaction between SIRT1 and 8-oxoguanine (8-oxoG)-DNA glycosylase 1 (OGG1) and/or insufficient interaction between the histone acetyltransferase p300 and OGG1 may decrease the acetylation of OGG1 in the lens of patients with ARC, resulting in abnormal accumulation of 8-oxoG, a biomarker of oxidative damage, in the lens (39). Eventually, these changes accelerate the development of ARC, implying a destructive role of SIRT1 upregulation (39). These divergent results are possibly attributed to the difference in research methods and subjects. More comprehensive studies focusing on the precise mechanisms of SIRT1 in the pathogenesis of $\mathrm{ARC}$ are required.

SIRT1 and age-associated macular degeneration. Ageassociated macular degeneration (AMD) manifests as either drusen/geographic atrophy or choroidal neovascularization (CNV). The pathophysiology and risk factors of AMD are complex (40). Chen et al (41) investigated three variants of the SIRT1 gene associated with AMD in Chinese Han individuals, and identified that the rs12778366 polymorphism within the promoter region of SIRT1 was significantly associated with AMD in recessive and codominant models. Expression of SIRT1 was more frequent in RPE and vascular endothelial cells (VECs) in human CNV membranes (42). By contrast, 
Deacetylase domain

\begin{tabular}{|l||l||c||c|c|}
\hline N-terminus & $\begin{array}{l}\text { Helical } \\
\text { module }\end{array}$ & $\begin{array}{c}\mathrm{Zn}^{2+} \\
\text { binding }\end{array}$ & $\begin{array}{c}\mathrm{NAD}^{+} \\
\text {binding }\end{array}$ & C-terminus \\
\hline \multicolumn{2}{|c|}{243} & 499
\end{tabular}

Figure 1. Schematic representation of the 1-dimensional structure of human SIRT1 protein. Human SIRT1 protein comprises 747 amino acids divided into three major regions: The central core, possessing the deacetylase domain, which consists of a large NAD ${ }^{+}$-binding subdomain and a smaller subdomain composed of a helical module and a $\mathrm{Zn}^{2+}$-binding module, and the $\mathrm{N}$ - and C-terminal domains, containing regulatory elements and binding domains for SIRT1 co-activators or repressors. SIRT, sirtuin.

another study indicated that the expression and self-renewal ability of SIRT1 in retinal stem cells (RSCs) (43), human retina and RPE cells (44) obviously declined with age.

Dysfunction of RPE cells is a major risk factor for the development of AMD. In aged RPE cells, overexpression of SIRT1 and octamer binding transcription factor 4, a POU-domain transcription factor, reprogrammed the cells into retinal progenitor-like cells and enhanced their antioxidant enzymatic activities (44). The expression of p53 increased in aged RPE, and in young RPE cells, sirtinol (a SIRT1 inhibitor) increased p53 acetylation and phosphorylation, but only had a marginal effect on p53 expression and increased caspase- 3 activation, which contributed to apoptosis of RPE cells (45). Furthermore, resveratrol obviously prevented p53 acetylation and phosphorylation, and eventually alleviated caspase-3-dependent RPE cell apoptosis (45). Recently, Golestaneh et al (46) developed an in vitro disease model of AMD through the generation of induced pluripotent stem cells (iPSCs) from RPE from patients with AMD and differentiation of these iPSCs into RPE (AMD RPE-iPSC-RPE), and observed the downregulation of SIRT1 and peroxisome proliferator activated receptor- $\gamma$ co-activator- $1 \alpha$ (PGC-1 $\alpha$ ) in AMD RPE-iPSC-RPE compared with that in normal RPE-iPSC-RPE. The study indicated that dysfunctional SIRT1/PGC-1 $\alpha$ may decrease mitochondrial activity and increase reactive oxygen species (ROS) production in AMD RPE-iPSC-RPE, and contribute to the pathophysiology of AMD (46).

Oxidative stress accelerates the progression of AMD. Overexpression of SIRT1 or treatment with resveratrol protected against oxidative stress-induced RPE cell senescence through downregulation of p53 K382 acetylation and $\mathrm{p} 21^{\text {Waf } 1 / \text { Cip } 1}$ accumulation (47), and increased the viability of $\mathrm{H}_{2} \mathrm{O}_{2}$-treated rat RSCs (43). On the contrary, knockdown of SIRT1 or application of SIRT1 inhibitors including nicotinamide (48) and sirtinol enhanced the toxicity of $\mathrm{H}_{2} \mathrm{O}_{2}$, making RPE cells hyper-sensitive to oxidative stress (47). Furthermore, SIRT1 rescued complement factor $\mathrm{H}(\mathrm{CFH})$ expression through increasing recruitment of signal transducer and activator of transcription 1 and decreasing the occupancy of the repressor FOXO3 in the CFH promoter of $\mathrm{H}_{2} \mathrm{O}_{2}$-treated ARPE-19 cells, which may prevent the oxidative stress-induced aging and cell damage and decrease the risk of AMD (49). A further experimental study indicated that SIRT1 levels were reduced in human RPE cells after treatment with amyloid $\beta(A \beta)$, which is one of the constituents of drusen (50). Treatment with SRT1720, a potent SIRT1 agonist that suppresses the nuclear factor (NF)- $\kappa B$ signaling system, significantly decreased $A \beta$-mediated upregulation of inflammatory cytokines in RPE cells, and balanced the morphology and barrier function of
RPE cell monolayers, which was obviously suppressed by knockdown of SIRT1 (50).

The expression of SIRT1 mRNA exhibited daily variations under the light-dark cycle conditions in the retina and was obviously upregulated in the dark phase (51). Considering that retinal cells consume more energy in the dark, the study linked SIRT1 regulation with the response to light stimuli and metabolic dysfunction in age-associated retinal diseases including AMD (51). In an in vitro study, ultraviolet B activated the phosphoinositide-3 kinase/AKT/extracellular signal-regulated kinase (ERK) pathway by reducing the expression of SIRT1 in a dose-dependent manner in ARPE-19 cells and suppressed the growth of the cells (52). In a mouse model of light-induced retinal degeneration, retinal SIRT1 activity was significantly reduced (53). Systemic administration of resveratrol not only significantly recovered retinal SIRT1 activity, but also restored histological and functional damage to the retina (53). Likewise, gene transfer of SIRT1 decreased retinal cell loss and improved the light-induced electroretinographic damage in rat retinas (44). In addition, the levels of activator protein (AP)-1 subunit c-fos were elevated in the retina of light-exposed mice and reduced by application of resveratrol (53). These studies suggest that SIRT1 activators or overexpression of SIRT1 protect the retina from light damage through inhibiting AP-1 bioactivity (53) and suppressing AKT and ERK phosphorylation (52).

CNV formation is a typical characteristic of wet AMD. Previous studies have indicated the regulative roles of SIRT1 in angiogenesis $(54,55)$. Expression of SIRT1 was higher in human CNV membranes from AMD patients than in eyes from donors without AMD (42). In vitro studies demonstrated that hypoxia-induced upregulation of SIRT1 levels augmented hypoxia-inducible factor (HIF)-2 $\alpha$ expression in choroidal endothelial cells, which in turn activated and released vascular endothelial growth factor (VEGF) (56). Thus, SIRT1 may promote CNV formation. However, other studies indicated that SIRT1 activation by resveratrol inhibited various inflammatory cytokines, transforming growth factor (TGF)- $\beta$-mediated VEGF secretion and hypoxia-mediated choroidal VEC proliferation through downregulation of HIF-1 $\alpha$ (57,58). A study by our group indicated that resveratrol inhibited the HIF-1 $\alpha / V E G F / V E G F$ receptor 2 signaling axis partly through SIRT1 (59). Khan et al (60) demonstrated that resveratrol inhibited the proliferation and migration of VECs and led to severely blunted neovascularization through activating eukaryotic elongation factor-2 kinase instead of the SIRT1-dependent pathway. The difference in drugs and experimental models may produce discrepant results regarding the function of SIRT1. The mechanisms of the effects of SIRT1 on CNV 


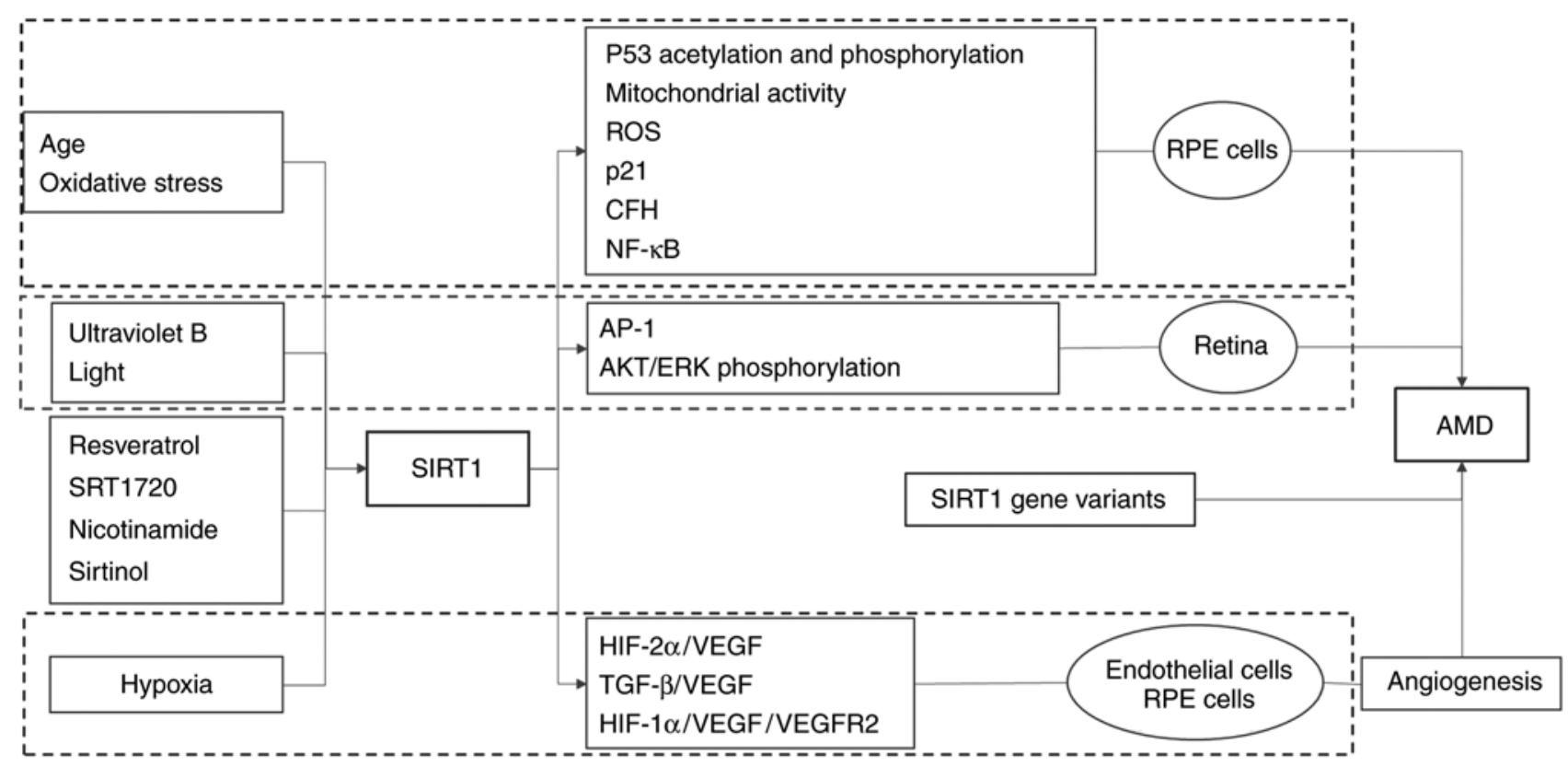

Figure 2. Role of SIRT1 in the pathogenesis of AMD. Factors and medicines (left) affected different cells and tissues function (right) through various pathways regulated by SIRT1 (middle), which ultimately induced the generation of AMD. SIRT, sirtuin; ROS, reactive oxygen species; RPE, retinal pigment epithelium; $\mathrm{NF}$, nuclear factor; AP, activator protein; ERK, extracellular signal-regulated kinase; HIF, hypoxia-inducible factor; VEGFR, vascular endothelial growth factor receptor; TGF, transforming growth factor; AMD, age-associated macular degeneration; $\mathrm{CFH}$, complement factor $\mathrm{H}$.

formation require more comprehensive elucidation. The role of SIRT1 in the pathogenesis of AMD is summarised in Fig. 2.

SIRT1 and diabetic retinopathy $(D R)$. DR is a severe complication of diabetes mellitus. Progression of diabetic blood glucose control suggests a 'metabolic memory' phenomenon $(61,62)$. The expression of SIRT1 was reduced in the retinas of diabetic mice (63-66). Zheng et al (67) observed a decrease in SIRT1 and an increase of NF- $\kappa \mathrm{B}$, the proapoptotic gene B-cell lymphoma 2-associated X protein (Bax), poly ADP-ribose polymerase (PARP) and ROS in bovine retinal endothelial cells (RECs) cultured under HG after glucose normalization. SIRT1 overexpression mediated liver kinase B1 (LKB1)/AMP-activated protein kinase (AMPK) activity, which inhibited ROS pathway activation in $\mathrm{HG}$ in RECs, resulting in the suppression of NF- $\kappa \mathrm{B}$, Bax and PARP expression. In addition, ROS-induced PARP activity, at least in part, led to the downregulation of SIRT1 expression and amplified an auto-feedback loop regulating SIRT1 expression. These results implied that SIRT1 mediated a metabolic memory effect induced by HG through the SIRT1/LKB1/AMPK/ROS cascade (67). Another study reported that transient hyperglycemia caused persistent endothelial cell senescence through the imbalance between SIRT1, and that P300 induced the upregulation of Ac-p53 and its downstream p21 (68). Recently, Zhao et al (69) identified that increased expression of miR-23b-3p directly downregulated SIRT1, which increased Ac-NF- $\kappa$ B levels in human RECs with a metabolic memory effect induced by HG. Similar results were obtained in rats with streptozotocin-induced diabetic retinopathy as a metabolic memory model, in which vascular permeability was significantly suppressed by miR-23b-3p inhibitor (69). Furthermore, metformin, a blood glucose-lowering therapeutic, fenofibrate, a lipid-lowering therapeutic and resveratrol suppressed the memory of hyperglycemic stress via the SIRT1-dependent signaling pathway $(67,68,70)$.

The overproduction of mitochondrial ROS and cytokines promotes the development of DR $(71,72)$. Reduced SIRT1 in HG-cultured RECs obviously enhanced the acetylation of $\mathrm{NF}-\kappa \mathrm{B}$ p 65 and AP-1, which binds to the promoter of matrix metalloproteinase (MMP)-9, which eventually activates MMP-9 (64,73). SIRT1 overexpression decreased the transcription of MMP-9 in REC $(64,73)$ and ameliorated $\mathrm{NF}-\kappa \mathrm{B} / \mathrm{Rac} 1 / \mathrm{NADPH}$ oxidase-mediated mitochondrial damage in diabetic rat retina (65). SIRT1 overexpression suppressed the upregulation of endothelin 1, TGF- $\beta 1$, collagen $1 \alpha$ and fibronectin, and prevented glucose-induced endothelial permeability and increases in total ROS/RNS levels in the retina of diabetic mice (74). In addition, systemic administration of resveratrol to the diabetic animals suppressed leukostasis and the upregulation of intercellular adhesion molecule-1 and VEGF (66). Exendin-4, a glucagon-like peptide 1 analogue, moderated ROS-mediated retinal cell death and recovered visual function by upregulating SIRT1 and SIRT3 expression in early-stage diabetic rats (75). Furthermore, SIRT1 may protect proliferative DR progression by inhibiting interleukin-17 (76). Another study indicated that miR-195 antagomir normalised tissue damage mediated by SIRT1 reduction in a rat model of DR (77).

Mice with oxygen-induced ischemic retinopathy (OIR) exhibit certain features of neovascularization that are characteristic of proliferative DR in humans (78). Increased SIRT1 in avascular retinal neurons of OIR mice mediated physiological revascularization of ischemic areas through modulating the HIF signaling pathway and secretion of pro-angiogenic and neuroprotective factors (79). However, ectopic overexpression of SIRT1 in mouse retinas or oral administration of SIRT1 activator did not alter the vaso-obliteration, pathologic 


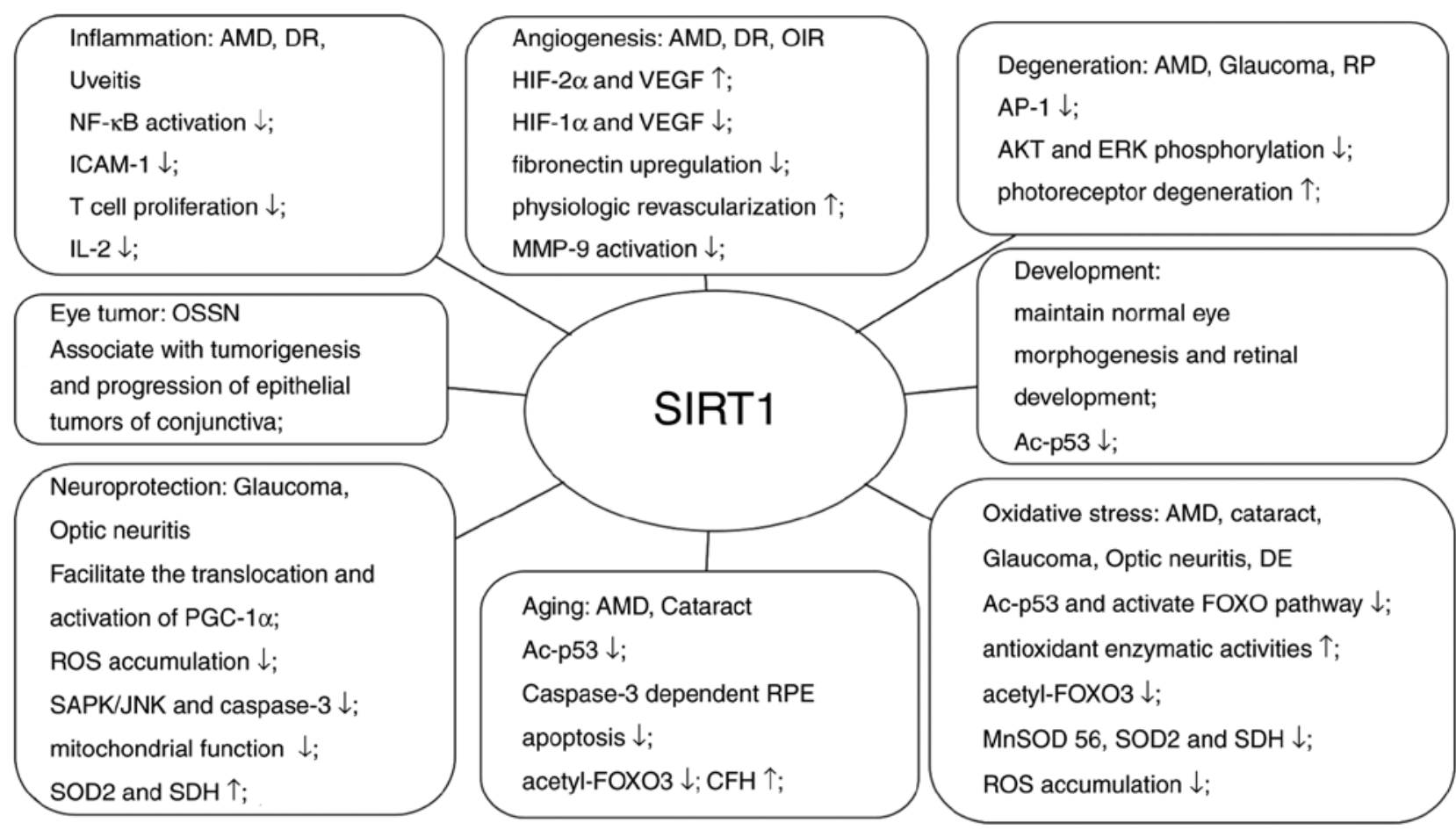

Figure 3. Roles of SIRT1 in eye diseases and development. The diagram depicts that SIRT1 plays significant role in ocular diseases by influencing various physiological and pathological processes such as inflammation, angiogenesis, aging, oxidative stress, neuroprotection and so on. $\downarrow$, suppression; $\uparrow$, enhancement. SIRT, sirtuin; AMD, age-associated macular degeneration; NF, nuclear factor; HIF, hypoxia-inducible factor; MMP, matrix metalloproteinase; SOD, superoxide dismutase; SDH, succinate dehydrogenase; JNK, c-Jun N-terminal kinase; ROS, reactive oxygen species; IL, interleukin; ICAM, intercellular adhesion molecule; AP, activator protein; ERK, extracellular signal-regulated kinase; Ac-p53, acetylated p53; CFH, complement factor H; FOXO, forkhead box O; RP, retinitis pigmentosa; RPE, retinal pigment epithelium; OSSN, ocular surface squamous neoplasia; OIR, oxygen-induced ischemic retinopathy; PGC, peroxisome proliferator-activated receptor $\gamma$ coactivator; SAPK, stress-activated protein kinase; DE, diabetic dry eye; DR, diabetic retinopathy.

neovascularization or retinal neuron degeneration in OIR (80). The protective role of SIRT1 in OIR requires more comprehensive study.

SIRT1 and glaucoma. Glaucoma is a group of chronic eye diseases ascribed to the irreversible death of retinal ganglion cells (RGCs) and progressive optic neuropathy, and results in serious vision loss and blindness. RGCs transmit light signals from the retina along their axons to the brain. Various types of stimuli, including trauma, ischemia, increased intraocular pressure, oxidative stress and inflammation, have been reported to lead to RGC death (81). SIRT1 has been linked with Alzheimer's and Huntington's disease in respective animal models and exerted a neuroprotective role in these diseases (82). Further studies have indicated the neuroprotective effects of SIRT1 on RGCs. For instance, resveratrol protected RGC-5 cells against serum deprivation-induced apoptosis by promoting the expression of SIRT1 and facilitating the translocation of PGC-1 $\alpha$ from the cytoplasm to the nucleus $(83,84)$. In addition, oral resveratrol administration or overexpression of SIRT1 following optic nerve crush injury in mice reduced RGC loss and ROS accumulation in the optic nerve (85). However, resveratrol was unable to prevent RGC loss after optic nerve crush injury in the eyes of SIRT1-knockout mice (85), which confirmed the necessity of SIRT1 expression for resveratrol-mediated neuroprotection. Furthermore, SIRT1 increased the viability of RGCs under hypoxic conditions through inhibiting stress-activated protein kinase/c-Jun $\mathrm{N}$-terminal kinase and caspase-3 activation (86), in ischemic mouse retinas, mangiferin prevented RGC loss via SIRT1, which was suppressed by sirtinol (87), suggesting a neuroprotection role of SIRT1 on RGCs under hypoxic condition.

SIRT1 and optic neuritis. RGC loss also has been demonstrated in several experimental models of optic neuritis, including experimental autoimmune encephalomyelitis (EAE), which is an animal model of multiple sclerosis (MS). SIRT1 activatorassociated suppression of RGCs loss delayed the onset of EAE and attenuated neuronal damage in EAE mice (88-90). The fact that the protective effect on RGCs by SIRT1 activators was blocked by sirtinol further suggests the neuroprotection role of SIRT1 activation $(88,90)$. Pre-treatment with SIRT1 activators, resveratrol and SRTAW04, significantly reduced ROS and cell death caused by $\mathrm{H}_{2} \mathrm{O}_{2}$ in RGC-5 cells (91). Furthermore, SIRT1 activators induced a significant increase in SOD2 and succinate dehydrogenase expression in stressed RGC-5 cells and enhanced deacetylation and activation of PGC-1 $\alpha$ (91). Similar protective mechanisms were observed in a mouse hepatitis virus A59-induced MS model (92). However, administration of SIRT1 activators neither suppressed the gross level of inflammation in the optic nerve nor attenuated the development of clinical EAE (88-90,92). During disease remission, EAE patients retain proper axonal density, suggesting that SIRT1 activator prevents permanent neurological dysfunction and neuronal damage in MS after acute spinal cord inflammation is resolved (90). In addition, resveratrol, through promoting SIRT1 expression and cholesterol synthesis, restored the number of surviving RGCs in the rats with optic nerve injury (93). Most 
importantly, the neuroprotective effects of SIRT1 activators without immunosuppression may imply a potential benefit of combining anti-inflammatory therapies for optic neuritis as well as for non-inflammatory optic nerve diseases.

SIRT1 and uveitis. Uveitis is characterized by a process of intraocular inflammation resulting from multiple factors. Corticosteroids and immunosuppressive drugs are effective for relieving diverse uveitis, but have severe side effects, which limits their clinical application (94). Alternative, novel drugs or treatments are therefore required. Recent studies have applied SIRT1 activators in the treatment of uveitis animal models. For instance, oral application of resveratrol to mice with endotoxin-induced uveitis (EIU) led to inhibition of oxidative damage and significant increases in SIRT1 activity in the RPE-choroid, resulting in the suppression of $\mathrm{NF}-\kappa \mathrm{B}-$ mediated inflammation in the eye (95). Resolvin D1, a lipid-derived protein for intravitreal injection, prevented EIU in rats through increasing SIRT1-mediated downregulation of Ac-p53 and FOXO1 (96). Furthermore, treatment of mice with experimental autoimmune uveoretinitis (EAU) with SIRT1 activator SRT2379 alleviated inflammation through suppressing $\mathrm{T}$ cell proliferation, pro-inflammatory cytokine production and leukocyte infiltration (97). Gardner et al (98) reported that tumor necrosis factor (TNF)- $\alpha$ mediated the cleavage and inactivation of SIRT1 to drain lymph node effector cells in EAU, and that combined application of a suboptimal TNF- $\alpha$ blockade and SIRT1 activation had a synergistic suppressive effect on EAU. In addition, in an in vitro model of antibody-mediated autoimmune retinopathy, resveratrol treatment led to an upregulation of SIRT1 and $\mathrm{Ku} 70$ in retinal cells, blocked the influx of intracellular calcium and the entry of pro-apoptotic Bax from the cytoplasm to the mitochondria, to subsequently prevent caspase-3 activation and protect cells from apoptotic death induced by antibodies against recoverin and $\alpha$-enolase (99). These studies demonstrated that activation of SIRT1 may be a potential treatment option for ocular uveitis.

\section{Perspective}

The present review mainly focused on the emerging evidence of the association between SIRT1 and the eyes. As summarized in Fig. 3, SIRT1 serves a significant role in ocular diseases by influencing various physiological and pathological processes such as inflammation, angiogenesis, aging, oxidative stress, neuroprotection. Although the potential protective role of SIRT1 has been demonstrated in numerous in vitro and in vivo models of ocular diseases, further studies are necessary to confirm the accurate mechanism and the most effective administration of SIRT1 activators and inhibitors in these diseases (100). In addition, it is required to determine whether the data obtained using animal models are applicable to human ocular diseases. In summary, SIRT1 may be considered as a valuable therapeutic target in ocular diseases.

\section{Acknowledgements}

The authors would like to thank Dr Yedi Zhou and Dr Lusi Zhang for their important advice regarding the preparation of the manuscript.

\section{Funding}

The present study was supported by the National Natural Science Foundation of China (grant no. 81703134), Department of Science and Technology, Hunan (grant no. 2015TP2007) and the Programs of Science-Technology Commission of Hunan province (grant no. 2015JC3036).

\section{Availability of data and materials}

The analysed data sets generated during the study are available from the corresponding author on reasonable request.

\section{Authors' contributions}

$\mathrm{MZ}$ and HZ drafted the article and revised it critically for important intellectual content. JL and HZ conceived and designed the present review.

\section{Ethical approval and consent to participate}

Not applicable.

\section{Consent for publication}

Not applicable.

\section{Competing interests}

The authors declare that they have no competing interests.

\section{References}

1. Portela A and Esteller M: Epigenetic modifications and human disease. Nat Biotechnol 28: 1057-1068, 2010.

2. Glozak MA, Sengupta N, Zhang X and Seto E: Acetylation and deacetylation of non-histone proteins. Gene 363: 15-23, 2005.

3. Gray SG and Ekström TJ: The human histone deacetylase family. Exp Cell Res 262: 75-83, 2001.

4. Gao L, Cueto MA, Asselbergs F and Atadja P: Cloning and functional characterization of HDAC11, a novel member of the human histone deacetylase family. J Biol Chem 277: 25748-25755, 2002.

5. Feldman JL, Dittenhafer-Reed KE and Denu JM: Sirtuin catalysis and regulation. J Biol Chem 287: 42419-42427, 2012.

6. Sauve AA, Wolberger C, Schramm VL and Boeke JD: The biochemistry of sirtuins. Annu Rev Biochem 75: 435-465, 2006.

7. Davenport AM, Huber FM and Hoelz A: Structural and functional analysis of human SIRT1. J Mol Biol 426: 526-541, 2014.

8. Yamakuchi M: MicroRNA regulation of SIRT1. Front Physiol 3: 68, 2012.

9. Sasaki T, Maier B, Koclega KD, Chruszcz M, Gluba W, Stukenberg PT, Minor W and Scrable H: Phosphorylation regulates SIRT1 function. PLoS One 3: e4020, 2008.

10. Yang Y, Fu W, Chen J, Olashaw N, Zhang X, Nicosia SV, Bhalla K and Bai W: SIRT1 sumoylation regulates its deacetylase activity and cellular response to genotoxic stress. Nat Cell Biol 9: 1253-1262, 2007.

11. Liu X, Wang D, Zhao Y, Tu B, Zheng Z, Wang L, Wang H, Gu W, Roeder RG and Zhu WG: Methyltransferase Set7/9 regulates p53 activity by interacting with Sirtuin 1 (SIRT1). Proc Natl Acad Sci USA 108: 1925-1930, 2011.

12. Kornberg MD, Sen N, Hara MR, Juluri KR, Nguyen JV, Snowman AM, Law L, Hester LD and Snyder SH: GAPDH mediates nitrosylation of nuclear proteins. Nat Cell Biol 12: 1094-1100, 2010.

13. Caito S, Rajendrasozhan S, Cook S, Chung S, Yao H, Friedman AE, Brookes PS and Rahman I: SIRT1 is a redox-sensitive deacetylase that is post-translationally modified by oxidants and carbonyl stress. FASEB J 24: 3145-3159, 2010. 
14. Tanno M, Sakamoto J, Miura T, Shimamoto K and Horio Y: Nucleocytoplasmic shuttling of the NAD ${ }^{+}$-dependent histone deacetylase SIRT1. J Biol Chem 282: 6823-6832, 2007.

15. Brachmann CB, Sherman JM, Devine SE, Cameron EE, Pillus L and Boeke JD: The SIR2 gene family, conserved from bacteria to humans, functions in silencing, cell cycle progression, and chromosome stability. Genes Dev 9: 2888-2902, 1995.

16. Guarente L: Sir2 links chromatin silencing, metabolism, and aging. Genes Dev 14: 1021-1026, 2000.

17. Ozawa Y, Kubota S, Narimatsu T, Yuki K, Koto T, Sasaki M and Tsubota K: Retinal aging and sirtuins. Ophthalmic Res 44: 199-203, 2010.

18. Mimura T, Kaji Y, Noma H, Funatsu H and Okamoto S: The role of SIRT1 in ocular aging. Exp Eye Res 116: 17-26, 2013.

19. Balaiya S, Abu-Amero KK, Kondkar AA and Chalam KV: Sirtuins expression and their role in retinal diseases. Oxid Med Cell Longev 2017: 3187594, 2017.

20. McBurney MW, Yang X, Jardine K, Hixon M, Boekelheide K, Webb JR, Lansdorp PM and Lemieux M: The mammalian SIR2alpha protein has a role in embryogenesis and gametogenesis. Mol Cell Biol 23: 38-54, 2003.

21. Kamel C, Abrol M, Jardine K, He X and McBurney MW: SirT1 fails to affect p53-mediated biological functions. Aging Cell 5: $81-88,2006$

22. Cheng HL, Mostoslavsky R, Saito S, Manis JP, Gu Y, Patel P, Bronson R, Appella E, Alt FW and Chua KF: Developmental defects and p53 hyperacetylation in Sir2 homolog (SIRT1)-deficient mice. Proc Natl Acad Sci USA 100 : 10794-10799, 2003

23. Chen D, Pacal M, Wenzel P, Knoepfler PS, Leone G and Bremner R: Division and apoptosis of E2f-deficient retinal progenitors. Nature 462: 925-929, 2009.

24. Jaliffa C, Ameqrane I, Dansault A, Leemput J, Vieira V, Lacassagne E, Provost A, Bigot K, Masson C, Menasche M and Abitbol M: Sirt1 involvement in rd10 mouse retinal degeneration. Invest Ophthalmol Vis Sci 50: 3562-3572, 2009.

25. Alves LF, Fernandes BF, Burnier JV, Mansure JJ, Maloney S, Odashiro AN, Antecka E, De Souza DF and Burnier MN Jr: Expression of SIRT1 in ocular surface squamous neoplasia Cornea 31: 817-819, 2012.

26. Maloney SC, Antecka E, Odashiro AN, Fernandes BF, Doyle M Lim LA, Katib YA and Miguel NB Jr: Expression of SIRT1 and DBC1 in developing and adult retinas. Stem Cells Int 2012: 908183, 2012.

27. Wang Y, Zhao X, Shi D, Chen P, Yu Y, Yang L and Xie L: Overexpression of SIRT1 promotes high glucose-attenuated corneal epithelial wound healing via p53 regulation of the IGFBP3/IGF-1R/AKT pathway. Invest Ophthalmol Vis Sci 54: 3806-3814, 2013

28. Liu H, Sheng M, Liu Y, Wang P, Chen Y, Chen L, Wang W and Li B: Expression of SIRT1 and oxidative stress in diabetic dry eye. Int J Clin Exp Pathol 8: 7644-7653, 2015.

29. An J, Chen X, Chen W, Liang R, Reinach PS, Yan D and Tu L: MicroRNA expression profile and the Role of miR-204 in corneal wound healing. Invest Ophthalmol Vis Sci 56: 3673-3683, 2015.

30. Gao J, Wang Y, Zhao X, Chen P and Xie L: MicroRNA-204$5 p$-mediated regulation of SIRT1 contributes to the delay of epithelial cell cycle traversal in diabetic corneas. Invest Ophthalmol Vis Sci 56: 1493-1504, 2015.

31. Wang Y, Zhao X, Wu X, Dai Y, Chen P and Xie L: microRNA-182 mediates Sirt1-induced diabetic corneal nerve regeneration. Diabetes 65: 2020-2031, 2016.

32. Hodge WG, Whitcher JP and Satariano W: Risk factors for age-related cataracts. Epidemiol Rev 17: 336-346, 1995.

33. Zheng Y, Liu Y, Ge J, Wang X, Liu L, Bu Z and Liu P: Resveratrol protects human lens epithelial cells against $\mathrm{H} 2 \mathrm{O} 2$-induced oxidative stress by increasing catalase, SOD-1, and HO-1 expression. Mol Vis 16: 1467-1474, 2010

34. Zheng T and Lu Y: SIRT1 protects human lens epithelial cells against oxidative stress by Inhibiting p53-dependent apoptosis. Curr Eye Res 41: 1068-1075, 2016.

35. Doganay S, Borazan M, Iraz M and Cigremis $\mathrm{Y}$ : The effect of resveratrol in experimental cataract model formed by sodium selenite. Curr Eye Res 31: 147-153, 2006.

36. Lin TJ, Peng CH, Chiou SH, Liu JH, Lin-Chung-Woung, Tsai CY, Chuang JH and Chen SJ: Severity of lens opacity, age, and correlation of the level of silent information regulator $\mathrm{T} 1$ expression in age-related cataract. J Cataract Refract Surg 37: $1270-1274,2011$
37. Zheng $\mathrm{T}$ and $\mathrm{Lu} \mathrm{Y}$ : Changes in SIRT1 expression and its downstream pathways in age-related cataract in humans. Curr Eye Res 36: 449-455, 2011

38. Kondo A, Goto M, Mimura T and Matsubara M: Silent information regulator $\mathrm{T} 1$ in aqueous humor of patients with cataract. Clin Ophthalmol 10: 307-312, 2016.

39. Kang L, Zhao W, Zhang G, Wu J and Guan H: Acetylated 8-oxoguanine DNA glycosylase 1 and its relationship with p300 and SIRT1 in lens epithelium cells from age-related cataract. Exp Eye Res 135: 102-108, 2015.

40. van Lookeren Campagne M, LeCouter J, Yaspan BL and Ye W: Mechanisms of age-related macular degeneration and therapeutic opportunities. J Pathol 232: 151-164, 2014.

41. Chen Z, Zhai Y, Zhang W, Teng Y and Yao K: Single nucleotide polymorphisms of the sirtuin 1 (SIRT1) gene are associated with age-related macular degeneration in Chinese han individuals: A case-control pilot study. Medicine (Baltimore) 94: e2238, 2015.

42. Maloney SC, Antecka E, Granner T, Fernandes B, Lim LA, Orellana ME and Burnier MN Jr: Expression of SIRT1 in choroidal neovascular membranes. Retina 33: 862-866, 2013.

43. Peng CH, Chang YL, Kao CL, Tseng LM, Wu CC, Chen YC, Tsai CY, Woung LC, Liu JH, Chiou SH and Chen SJ: SirT1-a sensor for monitoring self-renewal and aging process in retinal stem cells. Sensors 10: 6172-6194, 2010.

44. Peng CH, Cherng JY, Chiou GY, Chen YC, Chien CH, Kao CL, Chang YL, Chien Y, Chen LK, Liu JH, et al: Delivery of Oct4 and SirT1 with cationic polyurethanes-short branch PEI to aged retinal pigment epithelium. Biomaterials 32: 9077-9088, 2011.

45. Bhattacharya S, Chaum E, Johnson DA and Johnson LR: Age-related susceptibility to apoptosis in human retinal pigment epithelial cells is triggered by disruption of p53-Mdm2 association. Invest Ophthalmol Vis Sci 53: 8350-8366, 2012.

46. Golestaneh N, Chu Y, Cheng SK, Cao H, Poliakov E and Berinstein DM: Repressed SIRT1/PGC-1 $\alpha$ pathway and mitochondrial disintegration in iPSC-derived RPE disease model of age-related macular degeneration. J Transl Med 14: 344, 2016.

47. Zhuge CC, Xu JY, Zhang J, Li W, Li P, Li Z, Chen L, Liu X, Shang $\mathrm{P}, \mathrm{Xu} \mathrm{H}$, et al: Fullerenol protects retinal pigment epithelial cells from oxidative stress-induced premature senescence via activating SIRT1. Invest Ophthalmol Vis Sci 55: 4628-4638, 2014

48. Jackson MD, Schmidt MT, Oppenheimer NJ and Denu JM: Mechanism of nicotinamide inhibition and transglycosidation by Sir2 histone/protein deacetylases. J Biol Chem 278: 50985-50998, 2003.

49. Wu Z, Lauer TW, Sick A, Hackett SF and Campochiaro PA: Oxidative stress modulates complement factor $\mathrm{H}$ expression in retinal pigmented epithelial cells by acetylation of FOXO3. J Biol Chem 282: 22414-22425, 2007.

50. Cao L, Liu C, Wang F and Wang H: SIRT1 negatively regulates amyloid-beta-induced inflammation via the NF- $\kappa \mathrm{B}$ pathway. Braz J Med Biol Res 46: 659-669, 2013.

51. Ban N, Ozawa Y, Inaba T, Miyake S, Watanabe M, Shinmura K and Tsubota K: Light-dark condition regulates sirtuin mRNA levels in the retina. Exp Gerontol 48: 1212-1217, 2013

52. Chou WW, Chen KC, Wang YS, Wang JY, Liang CL and Juo SH: The role of SIRT1/AKT/ERK pathway in ultraviolet B induced damage on human retinal pigment epithelial cells. Toxicol In Vitro 27: 1728-1736, 2013.

53. Kubota S, Kurihara T, Ebinuma M, Kubota M, Yuki K, Sasaki M, Noda K, Ozawa Y, Oike Y, Ishida S and Tsubota K: Resveratrol prevents light-induced retinal degeneration via suppressing activator protein-1 activation. Am J Pathol 177: 1725-1731, 2010.

54. Potente M, Ghaeni L, Baldessari D, Mostoslavsky R, Rossig L, Dequiedt F, Haendeler J, Mione M, Dejana E, Alt FW, et al: SIRT1 controls endothelial angiogenic functions during vascular growth. Genes Dev 21: 2644-2658, 2007.

55. Potente M and Dimmeler S: Emerging roles of SIRT1 in vascular endothelial homeostasis. Cell Cycle 7: 2117-2122, 2008

56. Balaiya S, Khetpal V and Chalam KV: Hypoxia initiates sirtuin1-mediated vascular endothelial grow th factor activation in choroidal endothelial cells through hypoxia inducible factor- $2 \alpha$. Mol Vis 18: 114-120, 2012.

57. Nagineni CN, Raju R, Nagineni KK, Kommineni VK, Cherukuri A, Kutty RK, Hooks JJ and Detrick B: Resveratrol suppresses expression of VEGF by human retinal pigment epithelial cells: Potential nutraceutical for age-related macular degeneration. Aging Dis 5: 88-100, 2014.

58. Balaiya S, Murthy RK and Chalam KV: Resveratrol inhibits proliferation of hypoxic choroidal vascular endothelial cells. Mol Vis 19: 2385-2392, 2013. 
59. Zhang H, He S, Spee C, Ishikawa K and Hinton DR: SIRT1 mediated inhibition of VEGF/VEGFR2 signaling by resveratrol and its relevance to choroidal neovascularization. Cytokine 76: 549-552, 2015

60. Khan AA, Dace DS, Ryazanov AG, Kelly J and Apte RS Resveratrol regulates pathologic angiogenesis by a eukaryotic elongation factor-2 kinase-regulated pathway. Am J Pathol 177: 481-492, 2010.

61. Diabetes Control and Complications Trial Research Group; Nathan DM, Genuth S, Lachin J, Cleary P, Crofford O, Davis M, Rand $\mathrm{L}$ and Siebert C: The effect of intensive treatment of diabetes on the development and progression of long-term complications in insulin-dependent diabetes mellitus. N Engl J Med 329: 977-986, 1993.

62. Nathan DM, Cleary PA, Backlund JY, Genuth SM, Lachin JM Orchard TJ, Raskin P and Zinman B; Diabetes Control and Complications Trial/Epidemiology of Diabetes Interventions and Complications (DCCT/EDIC) Study Research Group: Intensive diabetes treatment and cardiovascular disease in patients with type 1 diabetes. N Engl J Med 353: 2643-2653, 2005.

63. Mortuza R, Chen S, Feng B, Sen S and Chakrabarti S: High glucose induced alteration of SIRTs in endothelial cells causes rapid aging in a p300 and FOXO regulated pathway. PLoS One 8: e54514, 2013

64. Kowluru RA, Santos JM and Zhong Q: Sirt1, a negative regulator of matrix metalloproteinase-9 in diabetic retinopathy. Invest Ophthalmol Vis Sci 55: 5653-5660, 2014.

65. Kowluru RA, Mishra M and Kumar B: Diabetic retinopathy and transcriptional regulation of a small molecular weight G-Protein, Rac1. Exp Eye Res 147: 72-77, 2016.

66. Kubota S, Ozawa Y, Kurihara T, Sasaki M, Yuki K, Miyake S, Noda K, Ishida S and Tsubota K: Roles of AMP-activated protein kinase in diabetes-induced retinal inflammation. Invest Ophthalmol Vis Sci 52: 9142-9148, 2011.

67. Zheng Z, Chen H, Li J, Li T, Zheng B, Zheng Y, Jin H, He Y, $\mathrm{Gu} \mathrm{Q}$ and $\mathrm{Xu} X$ : Sirtuin 1-mediated cellular metabolic memory of high glucose via the LKB1/AMPK/ROS pathway and therapeutic effects of metformin. Diabetes 61: 217-228, 2012.

68. Zhang E, Guo Q, Gao H, Xu R, Teng S and Wu Y: Metformin and resveratrol inhibited high glucose-induced metabolic memory of endothelial senescence through SIRT1/p300/p53/p21 pathway. PLoS One 10: e0143814, 2015.

69. Zhao S, Li T, Li J, Lu Q, Han C, Wang N, Qiu Q, Cao H, Xu X, Chen $\mathrm{H}$ and Zheng Z: miR-23b-3p induces the cellular metabolic memory of high glucose in diabetic retinopathy through a SIRT1-dependent signalling pathway. Diabetologia 59: 644-654, 2016.

70. Zhao S, Li J, Wang N, Zheng B, Li T, Gu Q, Xu X and Zheng Z: Fenofibrate suppresses cellular metabolic memory of high glucose in diabetic retinopathy via a sirtuin 1-dependent signalling pathway. Mol Med Rep 12: 6112-6118, 2015.

71. Simó $R$ and Hernández C: Novel approaches for treating diabetic retinopathy based on recent pathogenic evidence. Prog Retin Eye Res 48: 160-180, 2015.

72. Vujosevic S and Simó R: Local and systemic inflammatory biomarkers of diabetic retinopathy: An integrative approach. Invest Ophthalmol Vis Sci 58: BIO68-BIO75, 2017.

73. Mishra M, Flaga J and Kowluru RA: Molecular mechanism of transcriptional regulation of matrix metalloproteinase-9 in diabetic retinopathy. J Cell Physiol 231: 1709-1718, 2016.

74. Mortuza R, Feng B and Chakrabarti S: SIRT1 reduction causes renal and retinal injury in diabetes through endothelin 1 and transforming growth factor $\beta 1$. J Cell Mol Med 19: 1857-1867, 2015.

75. Zeng Y, Yang K, Wang F, Zhou L, Hu Y, Tang M, Zhang S, Jin S, Zhang J, Wang J, et al: The glucagon like peptide 1 analogue, exendin-4, attenuates oxidative stress-induced retinal cell death in early diabetic rats through promoting Sirt1 and Sirt3 expression. Exp Eye Res 151: 203-211, 2016.

76. Liu S, Lin YU and Liu XIN: Protective effects of SIRT1 in patients with proliferative diabetic retinopathy via the inhibition of IL-17 expression. Exp Ther Med 11: 257-262, 2016.

77. Mortuza R, Feng B and Chakrabarti S: miR-195 regulates SIRT1-mediated changes in diabetic retinopathy. Diabetologia 57: 1037-1046, 2014

78. Chen J and Smith LE: Retinopathy of prematurity. Angiogenesis 10 133-140, 2007.
79. Chen J, Michan S, Juan AM, Hurst CG, Hatton CJ, Pei DT, Joyal JS, Evans LP, Cui Z, Stahl A, et al: Neuronal sirtuin1 mediates retinal vascular regeneration in oxygen-induced ischemic retinopathy. Angiogenesis 16: 985-992, 2013.

80. Michan S, Juan AM, Hurst CG, Cui Z, Evans LP, Hatton CJ, Pei DT, Ju M, Sinclair DA, Smith LE and Chen J: Sirtuin1 over-expression does not impact retinal vascular and neuronal degeneration in a mouse model of oxygen-induced retinopathy. PLoS One 9: e85031, 2014

81. Fischer D and Leibinger M: Promoting optic nerve regeneration. Prog Retin Eye Res 31: 688-701, 2012.

82. Tang BL and Chua CE: SIRT1 and neuronal diseases. Mol Aspects Med 29: 187-200, 2008.

83. Kim SH, Park JH, Kim YJ and Park KH: The neuroprotective effect of resveratrol on retinal ganglion cells after optic nerve transection. Mol Vis 19: 1667-1676, 2013.

84. Chen S, Fan Q, Li A, Liao D, Ge J, Laties AM and Zhang X: Dynamic mobilization of PGC- $1 \alpha$ mediates mitochondrial biogenesis for the protection of RGC- 5 cells by resveratrol during serum deprivation. Apoptosis 18: 786-799, 2013.

85. Zuo L, Khan RS, Lee V, Dine K, Wu W and Shindler KS: SIRT1 promotes RGC survival and delays loss of function following optic nerve crush. Invest Ophthalmol Vis Sci 54: 5097-5102, 2013.

86. Balaiya S, Ferguson LR and Chalam KV: Evaluation of sirtuin role in neuroprotection of retinal ganglion cells in hypoxia. Invest Ophthalmol Vis Sci 53: 4315-4322, 2012.

87. Kim SJ, Sung MS, Heo H, Lee JH and Park SW: Mangiferin protects retinal ganglion cells in ischemic mouse retina via SIRT1. Curr Eye Res 41: 844-855, 2016.

88. Shindler KS, Ventura E, Rex TS, Elliott P and Rostami A: SIRT1 activation confers neuroprotection in experimental optic neuritis. Invest Ophthalmol Vis Sci 48: 3602-3609, 2007.

89. Fonseca-Kelly Z, Nassrallah M, Uribe J, Khan RS, Dine K, Dutt M and Shindler KS: Resveratrol neuroprotection in a chronic mouse model of multiple sclerosis. Front Neurol 3: 84, 2012.

90. Shindler KS, Ventura E, Dutt M, Elliott P, Fitzgerald DC and Rostami A: Oral resveratrol reduces neuronal damage in a model of multiple sclerosis. J Neuroophthalmol 30: 328-339, 2010.

91. Khan RS, Fonseca-Kelly Z, Callinan C, Zuo L, Sachdeva MM and Shindler KS: SIRT1 activating compounds reduce oxidative stress and prevent cell death in neuronal cells. Front Cell Neurosci 6: 63, 2012.

92. Khan RS, Dine K, Das Sarma J and Shindler KS: SIRT1 activating compounds reduce oxidative stress mediated neuronal loss in viral induced CNS demyelinating disease. Acta Neuropathol Commun 2: 3, 2014

93. Zhang Y, Li H, Cao Y, Zhang M and Wei S: Sirtuin 1 regulates lipid metabolism associated with optic nerve regeneration. Mol Med Rep 12: 6962-6968, 2015

94. Lin P, Suhler EB and Rosenbaum JT: The future of uveitis treatment. Ophthalmology 121: 365-376, 2014.

95. Kubota S, Kurihara T, Mochimaru H, Satofuka S, Noda K, Ozawa Y, Oike Y, Ishida S and Tsubota K: Prevention of ocular inflammation in endotoxin-induced uveitis with resveratrol by inhibiting oxidative damage and nuclear factor-kappaB activation. Invest Ophthalmol Vis Sci 50: 3512-3519, 2009.

96. Rossi S, Di Filippo C, Gesualdo C, Testa F, Trotta MC, Maisto R, Ferraro B, Ferraraccio F, Accardo M, Simonelli F and D'Amico M: Interplay between Intravitreal RvD1 and Local Endogenous Sirtuin-1 in the protection from endotoxin-induced uveitis in rats. Mediators Inflamm 2015: 126408, 2015

97. Gardner PJ, Joshi L, Lee RW, Dick AD, Adamson P and Calder VL: SIRT1 activation protects against autoimmune T cell-driven retinal disease in mice via inhibition of IL-2/Stat5 signaling. J Autoimmun 42: 117-129, 2013.

98. Gardner PJ, Yazid S, Chu CJ, Copland DA, Adamson P, Dick AD and Calder VL: TNF $\alpha$ regulates SIRT1 cleavage during ocular autoimmune disease. Am J Pathol 185: 1324-1333, 2015.

99. Anekonda TS and Adamus G: Resveratrol prevents antibody-induced apoptotic death of retinal cells through upregulation of Sirt1 and Ku70. BMC Res Notes 1: 122, 2008.

100. Bola C, Bartlett H and Eperjesi F: Resveratrol and the eye: Activity and molecular mechanisms. Graefes Arch Clin Exp Ophthalmol 252: 699-713, 2014. 